\title{
Plasma Apolipoproteins A1/B and OxLDL Levels in Patients with Covid-19 As Possible Markers of the Disease
}

\author{
V. V. Pushkarev ${ }^{a, *}$, L. K. Sokolova ${ }^{a, * *}$, S. A. Chervyakova ${ }^{a, * * *}$, Yu. B. Belchina ${ }^{a, * * * *}$, \\ O. I. Kovzun ${ }^{a, * * * * *}$, V. M. Pushkarev ${ }^{a, * * * * * *}$, and M. D. Tronko ${ }^{a, * * * * * * *}$ \\ a SI “V.P. Komisarenko Institute of Endocrinology and Metabolism, National Academy of Medical Sciences of Ukraine," \\ Kyiv, 04114 Ukraine \\ *e-mail: pushkarev.vm@gmail.com \\ **e-mail: liubov_sokolova@ukr.net \\ ***e-mail: svitlana.cherviakova@gmail.com \\ ****e-mail: belchina@ukr.net \\ *****e-mail:kovzun.oi@gmail.com \\ ******e-mail: endocrinology.kiev@gmail.com \\ *******e-mail: axolotle@gmail.com
}

Received June 23, 2021; revised July 24, 2021; accepted November 8, 2021

\begin{abstract}
The COVID-19 infection is associated with dyslipidemia and cardiovascular complications. The aim of the study was to determine the content of ApoA1, ApoB, and oxidized low-density lipoproteins (oxLDL) in the plasma of patients $(n=81)$ with COVID-19, diabetes, and cardiovascular disease (CVD). ApoA1, ApoB, and oxLDL were determined using enzyme-linked immunosorbent assay kits (Elabscience, United States). The measurements were performed at an optical wavelength of $450 \mathrm{~nm}$. It was shown that the level of ApoA1 in the blood of patients with type 2 diabetes and especially with COVID-19 was significantly lower than in the blood of healthy people. Blood ApoA1 levels did not show a further decrease in patients with both COVID-19 and diabetes or CVD compared to patients with COVID-19 without concomitant diseases. It was found that the level of ApoB in the blood of patients with diabetes and, especially, with COVID-19 is significantly higher than in the blood of healthy people. Blood levels of ApoB and oxLDL are higher in patients with both COVID-19 and diabetes or CVD compared to patients with COVID-19 without comorbidities. Thus, levels of ApoA1, ApoB, and oxLDL may be promising markers of COVID-19.
\end{abstract}

Keywords: COVID-19, diabetes mellitus, cardiovascular diseases, apolipoprotein A1, apolipoprotein B, oxLDL DOI: $10.3103 /$ S0095452721060116

\section{INTRODUCTION}

Hydrophobic lipids are insoluble in blood plasma and they are transported to the tissues as lipoproteins with cholesterol esters and triglycerides in the core and phospholipids, free cholesterol, and apolipoproteins on the surface. Elevated plasma levels of high-density lipoprotein (HDL-C) and apolipoprotein A1 (ApoAl) have been shown to be associated with a reduced risk of cardiovascular disease (CVD). In addition to the potential cardioprotective function, HDL and ApoA1, which is the major apoliproprotein of HDL, are also characterized by antidiabetic properties. Increased plasma HDL and ApoA1 improves glycemic control in patients with type 2 diabetes mellitus (DM2) by enhancing function of pancreatic $\beta$-cells and increasing insulin sensitivity (Rye, 2016). ApoAl also stimulates the glucose uptake in vivo in skeletal and cardiac muscles (Fritzen, 2020).
The hydrophobic core of low-density lipoproteins (LDL) consists of approximately 170 triglycerides, 1500 cholesterol esters, a hydrophilic shell consisting of 700 molecules of phospholipids, approximately 500 molecules of unesterified cholesterol, and one large ApoB with molecular weight of $500 \mathrm{kDa}$. ApoB is the major apolipoprotein and is a carrier for the following lipids: chylomicrons, LDL, very low-density lipoproteins (VLDL), intermediate-density lipoproteins (IDL), and lipoproteins (a). ApoB is not found in high-density lipoproteins (HDL), the latter being reduced to lipoproteins with ApoA. Hepatic ApoB has a molecular weight of $540000 \mathrm{Da}$. There are two circulating forms of ApoB - ApoB48 (from the small intestine) and ApoB100 (from the liver) (Devaraj, 2021).

Under oxidative stress, LDL oxidation occurs in the process of lipid peroxidation, mainly with the participation of phospholipid molecules. Under pathological conditions, plasma lipoproteins containing 


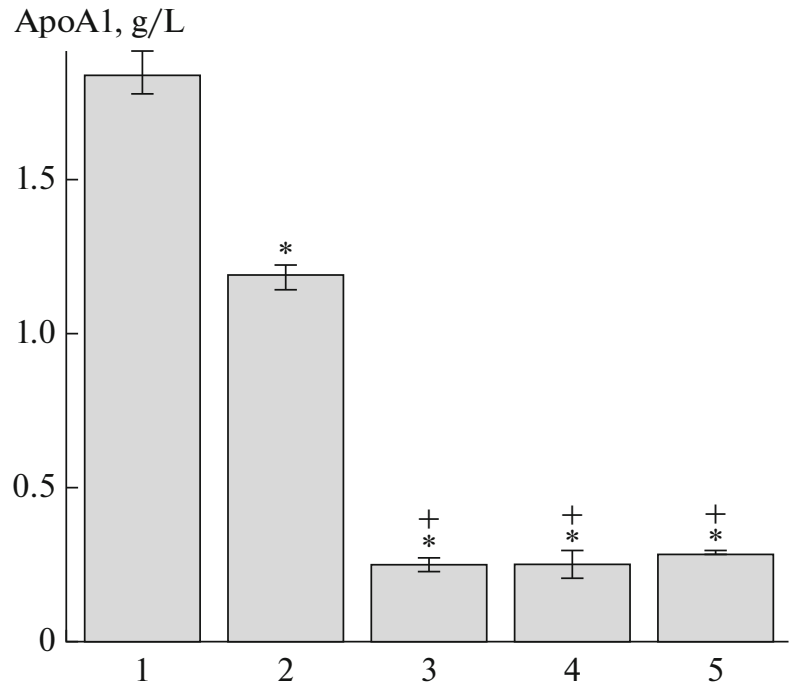

Fig. 1. Plasma ApoAl level in patients with diabetes and COVID-19 (g/L). 1-Control $(n=7) ; 2$-patients with $\mathrm{DM}(n=60) ; 3-$ patients with DM and COVID-19 $(n=16)$; 4-patients with COVID-19 $(n=5) ; 5$-patients with COVID-19 and CVD $(n=4) ; M \pm m . *$ Differences from control are significant, $P \ll 0.0001 ;+$ differences from group two are significant, $P \ll 0.0001$.

ApoB penetrate through the damaged endothelium into the subendothelial intima of blood vessels and oxidized by ROS (reactive oxygen species). Thus, LDL is modified to oxLDL (Khatana, 2020).

Elevated plasma levels of low-density lipoprotein cholesterol (LDL-C), apolipoprotein B (ApoB), and, especially, oxLDL have been shown to be associated with an increased risk of CVD. The ApoB/ApoA1 ratio is associated with DM2 and is proposed as a promising biomarker for CVD prediction (Mao, 2017). A meta-analysis also showed that elevated ApoB levels and decreased ApoAl levels, as well as the ApoB/Al ratio, are risk factors of a first ischemic stroke (Dong, 2015).

Since COVID-19 infection is associated with dyslipidemia and cardiovascular complications (Zamanian, 2020; Begue, 2021; Feingold, 2021; Kocar, 2021), the aim of the study was to determine the content of ApoA1, ApoB, and oxLDL in the plasma of patients with COVID-19, diabetes, and CVD.

\section{MATERIALS AND METHODS}

The study was conducted in the Diabetology Department of the Institute. The research protocol was approved by the Ethics Committee of the Institute. All patients signed an informed consent for further diagnostic and scientific research.

Blood was obtained by standard venipuncture and stored in EDTA tubes. Plasma was separated by centrifugation for 10 min after blood collection. The sam- ples were stored at $-80^{\circ} \mathrm{C}$ until use. The amount of ApoA1, ApoB, and oxLDL was determined $(n=81)$ using an enzyme-linked immunosorbent assay (ELISA) kit (Elabscience, United States). Measurements were performed at an optical wavelength of $450 \mathrm{~nm}$ using an enzyme-linked immunosorbent assay analyzer Stat Fax 3200 (Awareness Technology, United States).

Glycated hemoglobin was determined using one HbAlc FS kit (DiaSys Diagnostic Systems GmbH, Germany). The measurements were performed at an optical wavelength of $660 \mathrm{~nm}$.

Statistical analysis and data presentation were performed using the Origin 7.0 software. The results of the study are presented as $M \pm m$. Student's $t$-test was used for the comparison of data groups. Values of $P \leq$ 0.05 were considered significant.

\section{RESULTS AND DISCUSSION}

Blood plasma from 60 patients with diabetes (25 men, 35 women) and 21 patients with diabetes, CVD and COVID-19 (10 men, 11 women) was used. The blood from healthy people $(n=7)$ without comorbidities, representative of age, was used as the control. Four patients had a history of CVD. The level of $\mathrm{Hb} 1 \mathrm{Ac}$ in patients with diabetes was $9.62 \pm 0.27$ and BMI was $30.69 \pm 1.06 \mathrm{~kg} / \mathrm{m}^{2}$. The fasting blood glucose concentration of patients with COVID-19 and diabetes mellitus was $9.6 \pm 0.92 \mathrm{mM} / \mathrm{L}$; at the time of discharge $\mathrm{K} 6.72 \pm 0.62 \mathrm{mM} / \mathrm{L}$. The average $\mathrm{O}_{2}$ saturation was $87.3 \pm 0.7 \%$, indicating a severe course of the disease.

As can be seen in Fig. 1, the average level of ApoA1 in the blood from healthy people was in the upper range of the normal level $(1.88 \mathrm{~g} / \mathrm{L})$. In patients with diabetes, this value was much lower, $1.21 \mathrm{~g} / \mathrm{L}$, which is closer to the lower limit of normal. In patients with diabetes mellitus and COVID-19, the content of ApoA1 in the blood was approximately $0.25 \mathrm{~g} / \mathrm{L}$, which was more than four times less than the lower limit of normal content. Interestingly, differences between patients with COVID-19 and diabetes, COVID-19 and CVD, and patients with COVID-19 without comorbidities were not revealed (Fig. 1). In the blood from some patients with COVID-19, the level of ApoA1 decreased to almost zero: $0.09 \mathrm{~g} / \mathrm{L}$.

The fact that the level of ApoA1 in the blood was not reduced in patients with COVID-19 and diabetes and CVD compared with patients without comorbidities, suggests that COVID-19 forms a set of much more powerful factors affecting the content of ApoA1, and such a decrease reaches the lower limit.

Decreased levels of ApoA1 in the blood from patients with diabetes and who have an increased risk of CVD have been reported in many studies (Cochran, 2021; Gao, 2021; Retnakaran, 2019; Sokolova, 2014). COVID-19 significantly affected lipid profiles with a decrease in total cholesterol (TC), HDL-C and LDL-C 


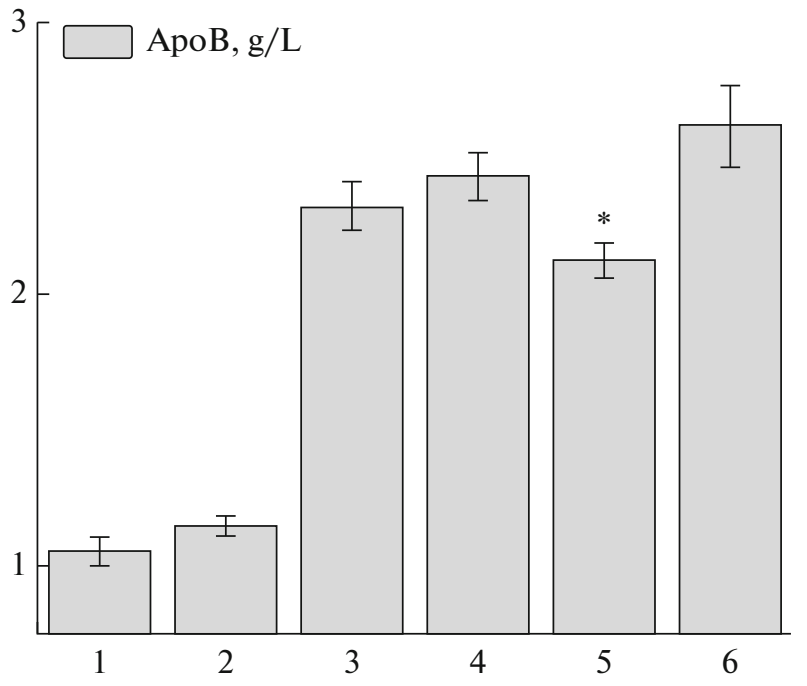

Fig. 2. Plasma ApoB level in patients with diabetes, CVD, and COVID-19. 1-Control $(n=7) ; 2$-patients with type 2 diabetes mellitus $(n=60) ; 3-$ mean data for all patients with COVID-19 $(n=21)$; 4 -patients with COVID-19 and diabetes $(n=16)$; 5-patients with COVID-19 without comorbidities $(n=5)$; 6-patients with COVID-19 and CVD $(n=4) ; M \pm m$. Significant differences between the control and other groups were revealed $(P<0.05$ for group two and $P \ll 0.0001$ for groups three to six); * differences from groups four and six were significant, $P<0.05$.

levels, and elevated triglycerides compared to controls. Plasma ApoA1 level in patients decreased by 55\% compared to healthy people (Begue, 2021). Hypolipidemia was observed in patients with COVID-19 and it was positively correlated with disease severity (Wei, 2020). ApoA1 is characterized by anti-inflammatory properties, is involved in the regulation of the immune response, and a decrease in the amount of this lipoprotein affects the severity of COVID-19. Moreover, dysregulation of ApoA1 may contribute to possible side effects of COVID-19 on the nervous system (Yang, 2020; Zamanian, 2020).

The reason for the decreased amount of plasma ApoA1 may be the inhibition of its synthesis in the liver and/or replacement by amyloid A in blood HDL (Begue, 2021). In addition, chymase produced by mast cells may play an important role in the degradation of ApoA1 (Inoue, 2020).

The average level of ApoB in the blood from healthy people was $1.05 \mathrm{~g} / \mathrm{L}$, the value was within the normal range (normal range: $0.55-1.3 \mathrm{~g} / \mathrm{L}$ for men and $0.6-1.4 \mathrm{~g} / \mathrm{L}$ for women) (Fig. 2). In patients with diabetes, this value was higher, $1.15 \mathrm{~g} / \mathrm{L}$, which was closer to the upper limit of normal range. In patients with COVID-19, the content of ApoB in the blood was much higher than the control and normal range values (Fig. 2, bars 3-6). The difference between patients with COVID-19 without comorbidities and COVID-

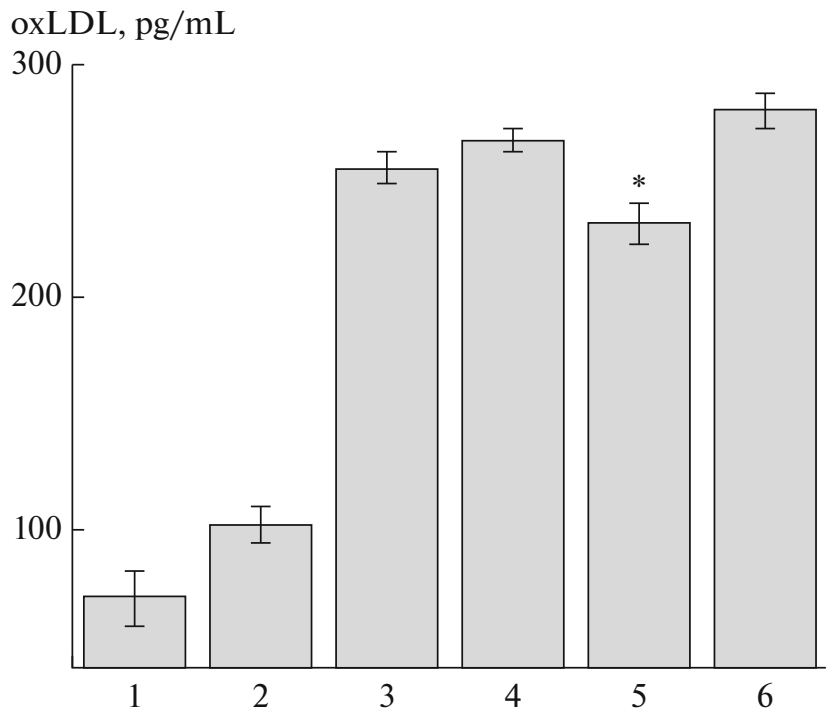

Fig. 3. Plasma oxLDL level in patients with diabetes, CVD, and COVID-19. 1-Control $(n=7) ; 2$-patients with type 2 diabetes mellitus $(n=60) ; 3$-average data for all patients with COVID-19 $(n=21)$; 4-patients with COVID-19 and diabetes $(n=16) ; 5$-patients with COVID-19 without comorbidities $(n=5) ; 6$-patients with COVID-19 and CVD $(n=4) . M \pm m$. Differences between the control and other groups were significant $(P<$ 0.05 for group two and $P \ll 0.0001$ for groups three to six); * differences from groups four and six were significant, $P<0.05$.

19 with diabetes and, especially, CVD should be noted (Fig. 1, bar 5 vs. bars 4 and 6).

A similar pattern was observed for oxLDL (Fig. 3). In patients with diabetes, the amount of oxLDL was above the control level. In patients with COVID-19, the content of oxLDL in the blood was much higher than the control level and the level in patients with diabetes (Fig. 3, bars 3-6). There was also a significant difference between patients with COVID-19 without comorbidities and COVID-19 with diabetes and CVD (Fig. 2, bar 5 vs. bars 4 and 6). Thus, the amount of ApoB and LDL derivatives, oxLDL, changed in the blood of patients with diabetes and patients with COVID-19 almost synchronously.

It has been proven that high LDL content causes the accumulation of cholesterol on the walls of blood vessels, significantly increasing the risk of atherosclerosis (AS) and CVD (Sniderman, 2019; Sokolova, 2017; Yan, 2020). Since ApoB is the major protein of LDL, the major transporter of cholesterol to cells, its concentration indicates the degree of risk of coronary heart disease. The concentration of ApoB in the blood is now considered a more reliable indicator of the risk of developing AS than total cholesterol (TC) or LDLcholesterol (LDL-C). Together with determining the concentration of $\mathrm{ApoB}$, the content of apolipoprotein A1 (ApoA1) should be determined. If the ratio of the concentration of ApoB to ApoA1 is high, then the risk of coronary heart disease is very high. It was proposed 
to set the normal range for ApoB to above $1.2 \mathrm{~g} / \mathrm{L}$. For risk groups, it was recommended to maintain an ApoB content at a level lower than $0.9 \mathrm{~g} / \mathrm{L}$ regardless of gender. The range limit values of the ApoB/ApoAl ratio can be used for the determination of the risk level for men $<0.9$ and for women $<0.8$ (Sniderman, 2019; Sokolova, 2014):

$\begin{array}{lc}\quad \text { Groups } & \text { ApoB/ApoA1 } \\ \text { Control } & 0.558 \\ \text { DM } & 0.952 \\ \text { Covid-19+ DM } & 9.041 \\ \text { Covid-19+ CVD } & 9.993\end{array}$

In the control group, this indicator was within the normal range, it was slightly higher than the normal range values in patients with diabetes, and it increased in patients with COVID-19 infection by an order of magnitude compared with patients with diabetes, indicating an extremely high risk of cardiovascular complications.

AS is characterized as a chronic inflammatory reaction to cholesterol deposition in the arteries. LDL, especially its oxidized form, oxLDL, play a crucial role in the onset and development of AS, inducing endothelial cell dysfunction, involving monocytes/macrophages, and contributing to chronic inflammation. Macrophages absorb oxLDL, forming foam cells that ultimately release proinflammatory cytokines and exacerbate local inflammation (Kocar, 2021; Yan, 2020).

We have shown that both ApoB and oxLDL levels increase with a high probability $\left(P=5.85 \times 10^{-8}\right.$ and $2.46 \times 10^{-11}$, respectively), indicating an increased risk of CVD.

SARS-related dyslipidemia has been confirmed. However, some authors found that serum TC, HDL, and LDL cholesterol levels were significantly lower in patients with COVID-19 compared to healthy people (Kocar, 2021; Wei, 2020). It has been concluded that patients with COVID-19 develop hypolipidemia when symptoms are mild and hypolipidemia worsen with the severity of the disease (Wei, 2020). However, the authors use the term "hypolipidemia" to refer to patients with total cholesterol $<174 \mathrm{mg} / \mathrm{dL}$. This definition of hypolipidemia is incorrect in the absence of a genetic diagnosis (Fogacci, 2020). The prevalence of patients with high cardiovascular risk may explain the observed LDL results. The low level of LDL cholesterol in this group was probably due to a more intense decrease in lipid levels as a result of therapy. In addition, we investigated the content of ApoB and not LDL-C, and they can have different dynamics. In blood samples obtained from patients with COVID-19, only the level of ApoA1was significantly reduced.

\section{CONCLUSIONS}

The level of plasma ApoA1 in patients with diabetes and, especially, COVID-19 was significantly lower than that in the blood of healthy people. The level of ApoB and oxLDL in the blood from patients with diabetes and, especially, COVID-19 was significantly higher than in the blood of healthy people. Differences between patients with COVID-19 without comorbidities and COVID-19 with diabetes or CVD were observed. ApoA1, ApoB, and oxLDL levels may be promising markers of COVID-19.

\section{FUNDING}

The article was prepared within the budget funding of the National Academy of Medical Ssciences of Ukraine according to the plan of research work of the State Institution Komisarenko Institute of Endocrinology and Metabolism of the National Academy of Medical Sciences of Ukraine.

\section{COMPLIANCE WITH ETHICAL STANDARDS}

Conflict of interest. The authors declare that they have no conflict of interests.

Statement on the welfare of animals. All procedures performed in studies involving human participants were in accordance with the ethical standards of the institutional and/or national research committee and with the 1964 Helsinki Declaration and its later amendments or comparable ethical standards. Informed consent was obtained from all individual participants involved in the study.

\section{REFERENCES}

Begue, F., Tanaka, S., Mouktadi, Z., et al., Altered highdensity lipoprotein composition and functions during severe COVID-19, Sci. Rep., 2021, vol. 11, no. 1, p. 2291. https://doi.org/10.1038/s41598-021-81638-1

Cochran, B.J., Ong, K.L., Manandhar, B., and Rye, K.A., High density lipoproteins and diabetes, Cells, 2021, vol. 10 , no. 4 , p. 850 . https://doi.org/10.3390/cells10040850

Devaraj, S., Semaan, J.R., and Jialal, I., Biochemistry, Apolipoprotein B, in StatPearls, Treasure Island, FL: StatPearls Publ., 2020.

Dong, H., Chen, W., Wang, X., et al., Apolipoprotein A1, B levels, and their ratio and the risk of a first stroke: a meta-analysis and case-control study, Metab. Brain Dis., 2015, vol. 30, no. 6, pp. 1319-1330. https://doi.org/10.1007/s11011-015-9732-7

Feingold, K.R., The bidirectional link between HDL and COVID-19 infections, J. Lipid Res., 2021, vol. 62, p. 100067. https://doi.org/10.1016/j.jlr.2021.100067

Fogacci, F., Borghi, C., and Cicero, A.F.G., Misinterpreting data in lipidology in the era of COVID-19, J. Clin. Lipidol., 2020, vol. 14, no. 4, pp. 543-544. https://doi.org/10.1016/j.jacl.2020.07.004

Fritzen, A.M., Domingo-Espin, J., Lundsgaard, A.M., et al., ApoA-1 improves glucose tolerance by increasing 
glucose uptake into heart and skeletal muscle independently of AMPKa2, Mol. Metab., 2020, vol. 35, p. 100949.

https://doi.org/10.1016/j.molmet.2020.01.013

Gao, L., Zhang, Y., Wang, X., and Dong, H., Association of apolipoproteins A1 and B with type 2 diabetes and fasting blood glucose: a cross-sectional study, BMC Endocrinol. Disord., 2021, vol. 21, no. 1, p. 59. https://doi.org/10.1186/s12902-021-00726-5

Inoue, Y., Okamoto, T., Honda, T., et al., Disruption in the balance between apolipoprotein A-I and mast cell chymase in chronic hypersensitivity pneumonitis, Immun. Inflamm. Dis., 2020, vol. 8, no. 4, pp. 659-671. https://doi.org/10.1002/ iid3.355

Khatana, C., Saini, N.K., Chakrabarti, S., et al., Mechanistic insights into the oxidized low-density lipoprotein-induced atherosclerosis, Oxid. Med. Cell Longev., 2020, vol. 2020, p. 5245308. https://doi.org/10.1155/2020/5245308

Kocar, E., Rezen, T., and Rozman, D., Cholesterol, lipoproteins, and COVID-19: basic concepts and clinical applications, Biochim. Biophys. Acta Mol. Cell Biol. Lipids, 2021, vol. 1866, no. 2, p. 158849. https://doi.org/10.1016/j.bbalip.2020.158849

Mao, Y., Xu, Y., and Lu, L., The nonlinear association between apolipoprotein B to apolipoprotein A1 ratio and type 2 diabetes, Medicine (Baltimore), 2017, vol. 96, no. 1, e5834. Erratum in: Medicine (Baltimore), vol. 96, no. 12, e6541. Erratum in: Medicine (Baltimore), vol. 96, no. 17 , no. e6821. https://doi.org/10.1097/MD.0000000000005834

Retnakaran, R., Ye, C., Connelly, P.W., et al., Serum apoAl (apolipoprotein A-1), insulin resistance, and the risk of gestational diabetes mellitus in human pregnancybrief report, Arterioscler. Thromb. Vasc. Biol., 2019, vol. 39, no. 10, pp. 2192-2197. https://doi.org/10.1161/ATVBAHA.119.313195

Rye, K.A., Barter, P.J., and Cochran, B.J., Apolipoprotein A-I interactions with insulin secretion and production,
Curr. Opin. Lipidol., 2016, vol. 27, no. 1, pp. 8-13. https://doi.org/10.1097/M0L.0000000000000253

Sniderman, A.D., Thanassoulis, G., Glavinovic, T., et al., Apolipoprotein B particles and cardiovascular disease: a narrative review, JAMA Cardiol., 2019, vol. 4, no. 12, pp. $1287-1295$.

https://doi.org/10.1001/jamacardio.2019.3780

Sokolova, L., Pushkarev, V., Pushkarev, V., et al., Diabetes mellitus and atherosclerosis. The role of inflammatory processes in pathogenesis, Int. J. Endocrinol. (Ukraine), 2017, vol. 13, no. 7, pp. 486-498. https://doi.org/10.22141/2224-0721.13.7.2017.115747

Sokolova, L.K., Risk factors and clinical and diagnostic aspects of cardiovascular pathology in patients with type 1 and 2 diabetes mellitus, Extended Abstract of Doctoral (Med.) Dissertation, Kyiv, Ukraine: Komisarenko State Institute of Endocrinology and Metabolism of the National Academy of Medical Sciences of Ukraine, 2014.

Wei, X., Zeng, W., Su, J., et al., Hypolipidemia is associated with the severity of COVID-19, J. Clin. Lipidol., 2020, vol. 14 , no. 3 , pp. $297-304$.

https://doi.org/10.1016/j.jacl.2020.04.008

Yan, Y., Song, D., Wu, J., and Wang, J., Long noncoding RNAs link oxidized low-density lipoprotein with the inflammatory response of macrophages in atherogenesis, Front. Immunol., 2020, vol. 11, p. 24. https://doi.org/10.3389/fimmu.2020.00024

Yang, Y., Zhu, Z., Fan, L., et al., Low serum level of apolipoprotein A1 is an indicator of severity in patients with coronavirus disease 2019, Preprint, 2020.

https://doi.org/10.21203/ rs.3.rs-31251/v1

Zamanian, A.M., Arjmand, B., Zali, A., and Razzaghi, M., Introducing AP0A1 as a key protein in COVID-19 infection: a bioinformatics approach, Gastroenterol. Hepatol. Bed Bench, 2020, vol. 13, no. 4, pp. 367-373.

Translated by V. Mittova 